SCIREA Journal of Mathematics http://www.scirea.org/journal/Mathematics

December 28, 2021

Volume 6, Issue 6, December 2021

https://doi.org/10.54647/mathematics11265

\title{
Determination of a time-dependent source term using local meshless method
}

\author{
Baiyu Wang *, Wei Liu \\ College of Computer Engineering and Applied Mathematics, Changsha University, China \\ *Corresponding author, Email:wangbaiyumath@hnu.edu.cn
}

\begin{abstract}
For the past few years, the meshless method has played a great advantage in solving partial differential equations. In this paper, a local meshless method based on moving least square and local radial basis functions is used to solve the inverse problem of heat conduction equation. The inverse problem is determination of a source term, and the unknown source term is time dependent. Numerical experiments are given to demonstrate the accuracy, effectiveness and feasibility of this method.
\end{abstract}

Keywords: Local meshless method, Inverse problem, Heat equation, Source term

\section{Introduction}

The inverse problem of heat conduction equation appears in a wide variety of physical problems, and the inverse source problem is one of the important class. There are various 
methods to deal with this kind of inverse problems.

$\mathrm{Liu}^{[1]}$ present an inverse problem using adjoint problem approach in nonlinear parabolic partial differential equation, and the applicability of this method is demonstrated in numerical examples.

Saadatmandi ${ }^{[2]}$ investigate the problem of simultaneous determination of the time dependent coefficients in one-dimensional partial differential equation applying tau technique. Hussein ${ }^{[3]}$ present the inverse problem of determining time and space dependent coefficients simultaneously in parabolic equations, the methods in that paper are finite-difference method and Tikhonov regularization method, and the influence of noisy data on the results is also studied. Fatullayev ${ }^{[4]}$ present the inverse problem of identification of an unknown source in heat equation, the method he used in the paper is approximating unknown function by polygons linear pieces, and the polygons linear pieces are determined consecutively from the solution of minimization problem based on the overspecified data. Le ${ }^{[5]}$ and Muhammad ${ }^{[6]}$ investigate the inverse source problem in the fractional differential equation using different methods.

In recent years, more and more researchers use meshless method to solve the inverse source problem of heat conduction equation.

Wang $^{[7]}$ investigate the numerical solution of a class of one-dimensional inverse parabolic problems using the moving least squares approximation, and the inverse problem is determination of an unknown source term depending on time. Yan ${ }^{[8]}$ and Amirfakhrian ${ }^{[9]}$ solve the inverse source problems using the meshless method. Farcas ${ }^{[10]}$ present two classes of inverse problems of determining unknown source term, one class is time dependent, and the other is space dependent.

Wang ${ }^{[11-12]}$ present a local meshless method based on the linear combination of moving least squares and local radial basis functions in the same compact support domain, by changing the coefficient of the linear combination, the new method possesses the properties of moving least squares approximation and local radial basis functions. Then the local meshless method is applied to find the numerical solutions of two classes of inverse problems in parabolic 
equations, one class is time dependent source term, the other class is time and space dependent.

In this paper, according to the ideas in [8], we use the local meshless method ${ }^{[11]}$ to solve a class of inverse heat source problem.

The outline of this paper is divided into the following sections: In Section 2, we introduce the local meshless method. In Section 3, we present the inverse source problem. In Section 4, we give the numerical solutions of the inverse problem. In Section 5, we present the numerical examples and discussions. Finally, we give the conclusion.

\section{The local meshless method}

Let $\Omega$ be an open bounded domain in $R^{d}$, given data values $x_{j}, u_{j}, j=1,2, \cdots, N$, where $x_{j}$ is the distinct scattered point in $\bar{\Omega}, \mathrm{u}_{\mathrm{j}}$ is the data value of function $u(x)$ at the node $x_{j}, N$ is the number of scattered nodes, and we let $\widetilde{u}(x)$ denote the approximate function of $u(x)$ in this paper.

Combining with the collocation method and the local meshless method in [11], we assume that the approximate function $\widetilde{u}$ can be written as

$$
\widetilde{u}(x)=\sum_{j=1}^{N} \zeta_{j}(x) u_{j}
$$

where $\zeta_{j}(x)$ stands for the shape function, and it can be written as the linear combination of some shape functions, which are composed of the moving least squares and the local radial basis functions,

$$
\zeta_{j}(x)=v \phi_{j}^{M}(x)+(1-v) \psi_{j}^{L}(x)
$$

where $\phi_{j}^{M}(x)$ and $\psi_{j}^{L}(x)$ stand for the shape functions in moving least squares and the local radial basis functions, respectively, $v$ is a constant which can be taken different values in $[0,1]$. 
Lemma $1^{[11]}$ Let $\Omega$ be an open and bounded domain in $R^{d}$, having a Lipschitz-continuous boundary, $u(x) \in H^{k}(\Omega),\left(k>\frac{d}{2}\right)$, then there exists a constant $h_{0}$, such that for all scattered points with $h \leq h_{0}$, we have

$$
\|u-\widetilde{u}\|_{H^{i}(\Omega)} \leq c h^{k-i}\|u\|_{H^{k}(\Omega)},(0 \leq i \leq k)
$$

where $h=\sup _{x \in \Omega} \min _{1 \leq j \leq N}\left\|x-x_{j}\right\|_{2}, \widetilde{u}$ is defined by (1), and $c$ is a constant independent of $h$.

\section{The inverse source problem}

Consider the following equation

$$
u_{t}(x, t)=a(x) u_{x x}(x, t)+f(t), x \in(0, l), t \in(0, T]
$$

with the initial condition

$$
u(x, 0)=u_{0}(x), x \in(0, l)
$$

the boundary conditions

$$
u(0, t)=p(t), u(l, t)=q(t), t \in(0, T],
$$

and the additional observation of $u(x, t)$ at some internal point $x_{0}, x_{0} \in(0, l)$,

$$
u\left(x_{0}, t\right)=E(t), t \in(0, T] .
$$

In above formulas (3)-(6), the function $f(t)$ is unknown, so the problem is inverse source problem, according to the ideas of Yan ${ }^{[8]}$, let

$$
r(t)=\int_{0}^{t} f(\xi) d \xi, \quad v(x, t)=u(x, t)-r(t)
$$

from (7) and (3)-(6), we get

$$
v_{t}(x, t)=a(x) v_{x x}(x, t), x \in(0, l), t \in(0, T],
$$

with the initial condition

$$
v(x, 0)=u_{0}(x), x \in(0, l)
$$

and the boundary conditions 


$$
v(0, t)=p(t)-r(t), v(l, t)=q(t)-r(t), t \in(0, T],
$$

from (6) and (7), we get

$$
v\left(x_{0}, t\right)=E(t)-r(t), t \in(0, T]
$$

combine with (10) and (11), we have

$$
v(0, t)-v\left(x_{0}, t\right)=p(t)-E(t), v(l, t)-v\left(x_{0}, t\right)=q(t)-E(t), t \in(0, T]
$$

Through the above derivation process, the inverse problem is transformed to a direct problem, then we use the local meshless method described in section 2 solving the problem (8),(9) and (12).

\section{The numerical solutions}

When the collocation method is employed in conjunction with the local meshless method to solve the equations (8),(9) and (12), the exact solution $v(x, t)$ is replaced by the approximate solution $\tilde{v}(x, t)$. We followed the method of Wang ${ }^{[11]}$, and according to (1), at the time $t=$ $t_{m}\left(m=1,2, \cdots, M, 0=t_{1}<t_{2}<\cdots<t_{M}=T\right), \tilde{v}\left(x, t_{m}\right)$ can be represented as

$$
\tilde{v}\left(x, t_{m}\right)=\sum_{j=1}^{N} \zeta_{j}(x) \tilde{v}\left(x_{j}, t_{m}\right)
$$

where $\zeta_{j}(x)$ is the shape function described in section $2, \tilde{v}\left(x_{j}, t_{m}\right)$ is the value of the approximate function $\tilde{v}(x, t)$ at $x_{j}$ and $t_{m}$.

Then, we have

$$
\tilde{v}_{x x}\left(x, t_{m}\right)=\sum_{j=1}^{N} \zeta_{j}^{\prime \prime}(x) \tilde{v}\left(x_{j}, t_{m}\right)
$$

for the time derivative $\tilde{v}_{t}\left(x, t_{m}\right)$, we apply one step forward difference formula,

$$
\tilde{v}_{t}\left(x, t_{m}\right)=\frac{\tilde{v}\left(x, t_{m+1}\right)-\tilde{v}\left(x, t_{m}\right)}{\Delta t}
$$

where $\Delta t=t_{m+1}-t_{m}, m=1,2, \cdots, M-1$.

So for $t=t_{m}$, the equation (8) can be rewritten as 


$$
\frac{\tilde{v}\left(x, t_{m+1}\right)-\tilde{v}\left(x, t_{m}\right)}{\Delta t}=a(x) \sum_{j=1}^{N} \zeta_{j}^{\prime \prime}(x) \tilde{v}\left(x_{j}, t_{m}\right)
$$

that is equivalent to

$$
\tilde{v}\left(x, t_{m+1}\right)=\tilde{v}\left(x, t_{m}\right)+\Delta t \cdot a(x) \sum_{j=1}^{N} \zeta_{j}^{\prime \prime}(x) \tilde{v}\left(x_{j}, t_{m}\right)
$$

by the collocation method, we select $N$ distinct points in $\Omega$, and substitute each $x_{k}(k=$ $1,2, \cdots, N)$ for $x$ in $(17)$, then we get

$$
\tilde{v}\left(x_{k}, t_{m+1}\right)=\tilde{v}\left(x_{k}, t_{m}\right)+\Delta t \cdot a\left(x_{k}\right) \sum_{j=1}^{N} \zeta_{j}^{\prime \prime}\left(x_{k}\right) \tilde{v}\left(x_{j}, t_{m}\right)
$$

Combining with the conditions (9) and (12), for the iterative system of equation (18), after computation using the iterative method, we can obtain the numerical solution

$$
\tilde{v}\left(x_{k}, t_{m}\right)(k=1,2, \cdots, N, m=1,2, \cdots, M)
$$

\section{Numerical results and discussions}

In this section, we give examples to illustrate the accuracy, feasibility and effectiveness of the method.

Example 1. Consider the problems (3)-(6) with the conditions

$$
\begin{gathered}
u_{0}(x)=2+\sin x, p(t)=(2+t) e^{-t}, q(t)=(2+t+\sin l) e^{-t}, \\
E(t)=\left(2+t+\sin x_{0}\right) e^{-t}
\end{gathered}
$$

The exact solutions are

$$
u(x, t)=(2+t+\sin x) e^{-t}, f(t)=(-1-t) e^{-t}
$$

and $T=2, l=2, x_{0}=1$.

Firstly, we give the comparisons of the exact solutions $f(t), u(x, t)$ and the numerical solutions $\tilde{f}(t), \widetilde{u}(x, t)$ at some nodes.

Table 1 the comparisons of the exact and numerical solutions

\begin{tabular}{lllllll}
$t$ & $f(t)$ & $\tilde{f}(t)$ & $u(0.5, t)$ & $\widetilde{u}(0.5, t)$ & $u(1.75, t)$ & $\widetilde{u}(1.75, t)$ \\
\hline
\end{tabular}




\begin{tabular}{lllllll}
\hline 0.2 & -0.9825 & -0.9825 & 2.1937 & 2.1937 & 2.6068 & 2.6068 \\
0.4 & -0.9385 & -0.9385 & 1.9301 & 1.9301 & 2.2684 & 2.2684 \\
0.6 & -0.8781 & -0.8781 & 1.6900 & 1.6900 & 1.9669 & 1.9669 \\
0.8 & -0.8088 & -0.8088 & 1.4735 & 1.4735 & 1.7003 & 1.7003 \\
1.0 & -0.7358 & -0.7358 & 1.2800 & 1.2800 & 1.4656 & 1.4656 \\
1.2 & -0.6626 & -0.6626 & 1.1082 & 1.1082 & 1.2602 & 1.2602 \\
1.4 & -0.5918 & -0.5918 & 0.9567 & 0.9567 & 1.0811 & 1.0811 \\
1.6 & -0.5249 & -0.5249 & 0.8324 & 0.8324 & 0.9255 & 0.9255 \\
1.8 & -0.4628 & -0.4629 & 0.7074 & 0.7074 & 0.7908 & 0.7908 \\
\hline
\end{tabular}

From Table 1, we see that the exact solutions are approximately identical in the numerical solutions at different nodes, and the maximum error is not more than $1 \times 10^{-4}$, so the approximation effect is very good.

Secondly, in order to illustrate the numerical accuracy of the method in this paper, let

$$
\operatorname{err} f(t)=f(t)-\tilde{f}(t), \operatorname{erru}(x, t)=u(x, t)-\tilde{u}(x, t),
$$

we plot the exact solution $f(t)$, the numerical solution $\tilde{f}(t)$ and the error function $\operatorname{err} f(t)$ for different $\Delta t$ in Figures 1-3, and we plot the exact solution $u(x, t)$, the numerical solution $\widetilde{u}(x, t)$ and the error function $\operatorname{erru}(x, t)$ for different $\Delta t$ in Figures 4-5.
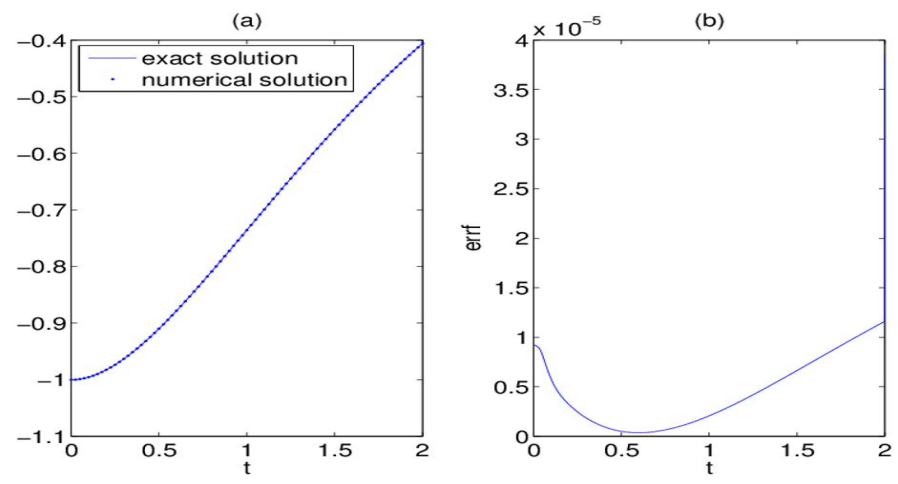

Figure $1 \Delta t=0.0001,(a): f(t)$ and $\tilde{f}(t),(b)$ :the error function errf $(t)$. 
(a)

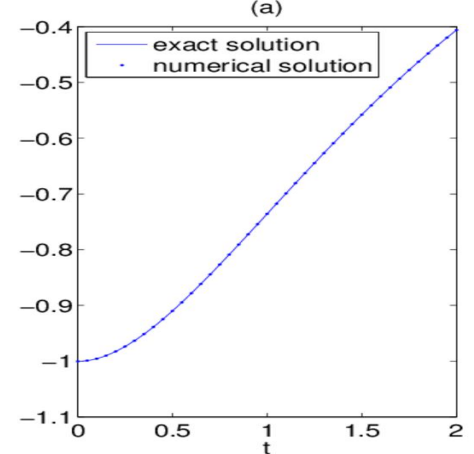

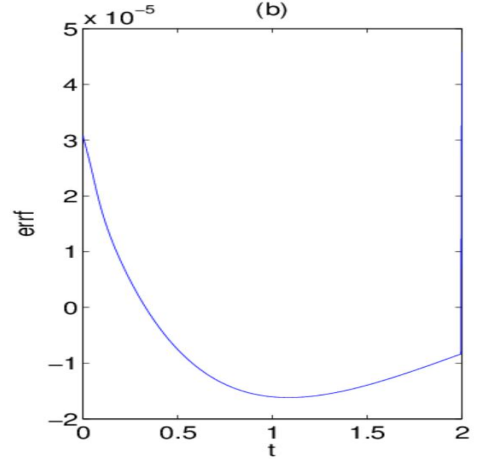

Figure $2 \Delta t=0.0002,(a): f(t)$ and $\tilde{f}(t),(b)$ :the error function $\operatorname{err} f(t)$.
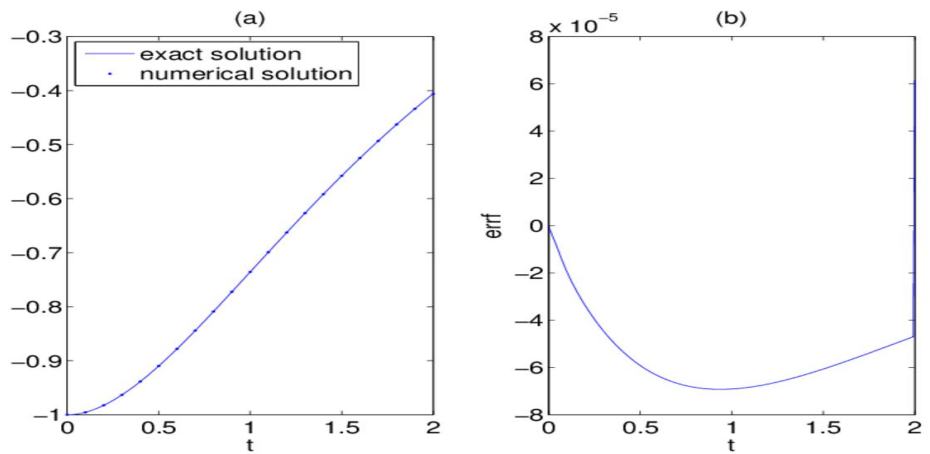

Figure $3 \Delta t=0.0004,(a): f(t)$ and $\tilde{f}(t),(b)$ :the error function $\operatorname{err} f(t)$.

(a)

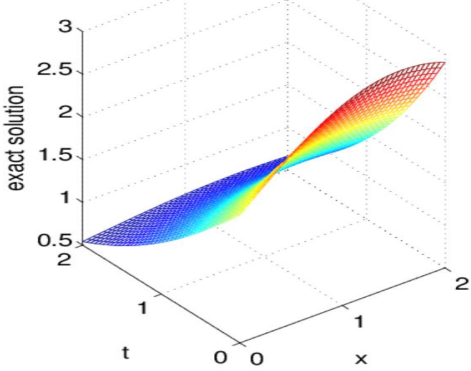

(b)

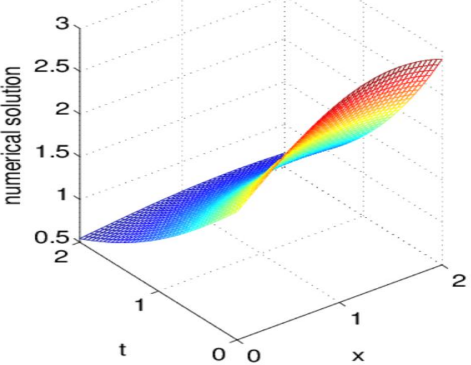

Figure $4(a): u(x, t),(b): \widetilde{u}(x, t)$. 

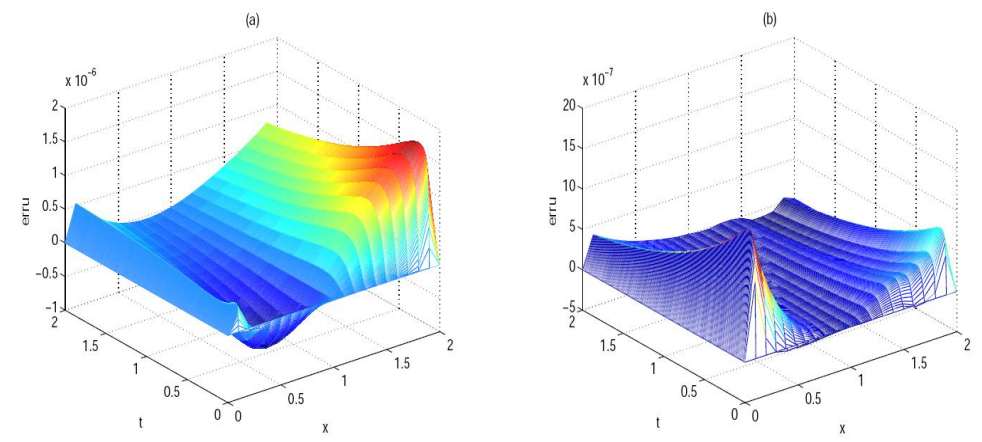

Figure 5 erru $(x, t)$ for different $\Delta t,(a): \Delta t=0.0001,(b): \Delta t=0.0004$.

For purpose of observing the effect of the approximation more clearly, we plot the function $u(x, t)$ at $x=1.5$ and $t=0.8$, respectively, in Figure 6.
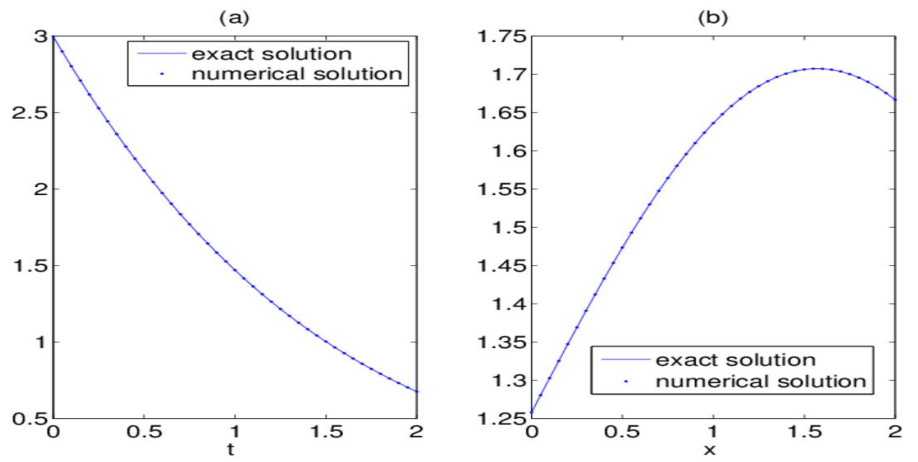

Figure $6 \quad(a): u(1.5, t), \quad(b): u(x, 0.8)$.

From the above six figures, we see intuitively that the numerical solutions are close to the exact solutions, and the effect of the approximation is good.

Thirdly, in order to study the influence of the noise on the numerical results, we define the observed data with noise disturbance as

$$
E_{\gamma}(t)=E(t)(1+\gamma)
$$

where $\gamma$ represents the noise data.

We plot the exact solution $f(t)$ and the numerical solution $\tilde{f}(t)$ with different noise data $\gamma$ in Figure 7, the error functions $\operatorname{err} f(t)$ and $\operatorname{erru}(x, t)$ with different noise data $\gamma$ in Figures $8-9$. 

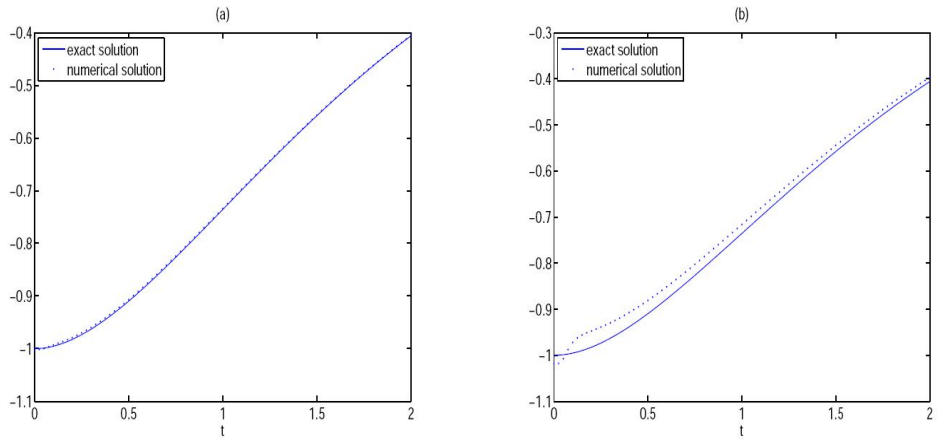

Figure $7 f(t)$ and $\tilde{f}(t)$ for different $\gamma,(a): \gamma=0.001,(b): \gamma=0.01$
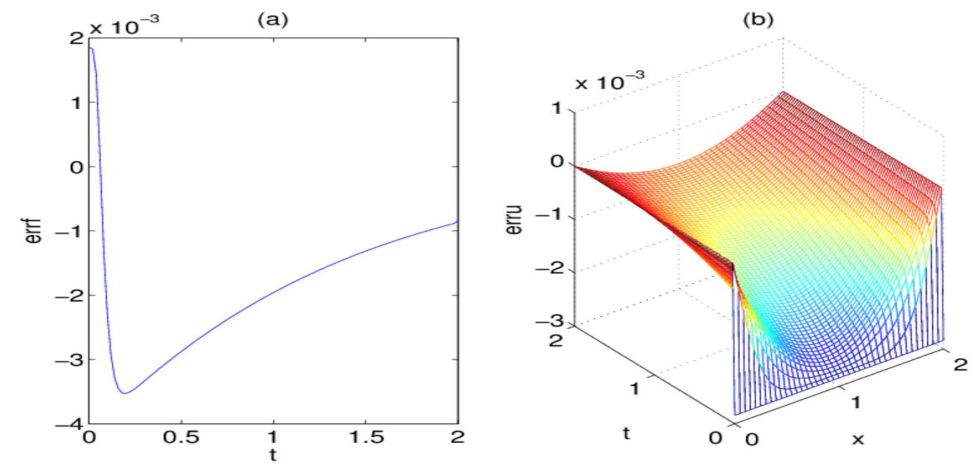

Figure $8 \quad \gamma=0.001(a): \operatorname{err} f(t),(b): \operatorname{erru}(x, t)$.
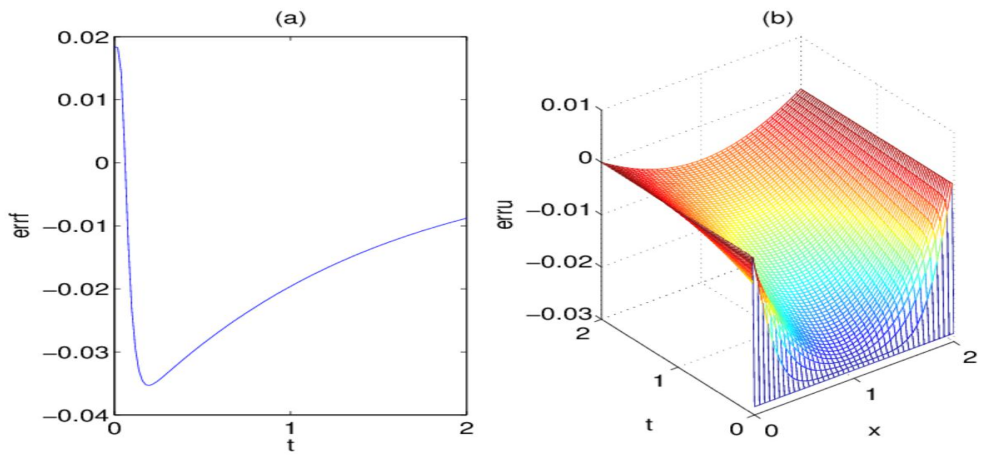

Figure $9 \gamma=0.01(a): \operatorname{err} f(t),(b): \operatorname{erru}(x, t)$.

From the above three figures, we get that when the noise parameter is large, the approximation effect is relatively poor, but there is no obvious oscillation, and the error decreases with the decrease of noise parameter.

Finally, in order to test the feasibility of the numerical method, and to get a better view of the approximation, we define the errors of $f(t)$ and $u(x, t)$ as follows 


$$
\begin{gathered}
\operatorname{Ref}=\sqrt{\frac{1}{N} \sum_{j=1}^{N}\left(f\left(t_{j}\right)-\tilde{f}\left(t_{j}\right)\right)^{2}}, \\
\operatorname{Reu}=\sqrt{\frac{1}{M N} \sum_{i=1}^{M} \sum_{j=1}^{N}\left(u\left(x_{i}, t_{j}\right)-\tilde{u}\left(x_{i}, t_{j}\right)\right)^{2}},
\end{gathered}
$$

we give the results of Ref and Reu for different $\Delta t$ with different methods in Table 2, where MLS represents the moving least squares approximation method, LRBF represents the local radial basis function method, and LMM represents the method in this paper.

Table 2 Results for different $\Delta t$ with different methods

\begin{tabular}{cccc}
\hline & & Ref & Reu \\
\hline \multirow{3}{*}{$\Delta t=0.0001$} & MLS & $6.9733 \times 10^{-5}$ & $2.7469 \times 10^{-6}$ \\
& LRBF & $5.1160 \times 10^{-6}$ & $6.5810 \times 10^{-7}$ \\
& LMM & $5.5952 \times 10^{-6}$ & $5.6554 \times 10^{-7}$ \\
\hline \multirow{2}{*}{$\Delta t=0.0002$} & MLS & $4.3674 \times 10^{-5}$ & $2.1959 \times 10^{-6}$ \\
& LRBF & $3.1415 \times 10^{-5}$ & $1.1154 \times 10^{-6}$ \\
& LMM & $1.3323 \times 10^{-5}$ & $4.5712 \times 10^{-7}$ \\
\hline \multirow{2}{*}{$\Delta t=0.0004$} & MLS & $4.7692 \times 10^{-5}$ & $1.1069 \times 10^{-6}$ \\
& LRBF & $9.5845 \times 10^{-5}$ & $2.1718 \times 10^{-6}$ \\
& LMM & $5.7599 \times 10^{-5}$ & $3.3496 \times 10^{-7}$ \\
\hline
\end{tabular}

As indicated in Table 2, we get that as the decease of $\Delta t$, the value of Ref and Reu are decrease too, and the results obtained by the method in this paper are superior to those obtained by other two methods, so the method in this paper is feasible.

\section{Conclusion}

In this paper, we use a local meshless method to solve the inverse source problem of heat conduction. Numerical experiments demonstrate that the method in this paper is accurate, feasible and effective. 


\section{Acknowledgment}

This work was supported by Scientific Research Fund of Scientific and Technological Project of Changsha City, (No. kc1809021), and Scientific Research Fund of Hunan Provincial Education Department (No. 18B419).

\section{References}

[1] LIU Z.H., WANG B.Y., Coefficient Identification in Parabolic Equations. Applied Mathematics and Computation, 209,379-390,2009.

[2] SAADATMANDI A., DEHGHAN M., Computation of Two-Dependent Coefficients in a Parabolic Partial Differential Equation Subject to Additional Specifications. International Journal of Computer Mathematics , 87,997-1008,2010.

[3] HUSSEIN M. S., LESNIC D., Simultaneous Determination of Time and Space-Dependent Coefficients in a Parabolic Equation. Communications in Nonlinear Science and Numerical Simulation , 33,194-217,2016.

[4] FATUllayeV A. G., Numerical Solution of the Inverse Problem of Determining an Unknown Source Term in a Heat Equation. Mathematics and Computers in Simulation, $58,247-253,2002$.

[5] LE DINH LONG, ZHOU Y., TRAN THANA BINH and NGUYEN CAN., A Mollification Regularization Method for the Inverse Source Problem for a Time Fractional Diffusion Equation. Mathematics , 7, 1048; doi:10.3390/math7111048,2019.

[6] MUHAMMAD ALI, SARA AZIZ, SALMAN A MALIK., Inverse source problems for a space-time fractional differential equation. Inverse Problems in Science and Engineering, 28(1),47-68,2019.

[7] WANG B.Y., Moving Least Squares Method for a One-Dimensional Parabolic Inverse Problem. Abstract and Applied Analysis, Article ID 686020, 12 pages,2014.

[8] YAN L., FU C.L., YANG F.L., The method of fundamental solutions for the inverse heat source problem. Engineering Analysis with Boundary Elements , 32,216-222,2008.

[9] AMIRFAKHRIAN M., ARGHAND M., KANSA E. J., A new approximate method for an 
inverse time-dependent heat source problem using fundamental solutions and RBFs. Engineering Analysis with Boundary Elements , 64,278-289,2016.

[10] FARCAS A., LESNIC D., The Boundary-Element Method for the Determination of a Heat Source Dependent on One Variable. Journal of Engineering Mathematics , $54,375-388,2006$.

[11] WANG B.Y., A Local Meshless Method Based on Moving Least Squares and Local Radial Basis Functions. Engineering Analysis with Boundary Elements , $50,395-401,2015$.

[12] LIU W., WANG B.Y., A local meshless method for two classes of parabolic inverse problems. Journal of Applied Mathematics and Physics , 6,968-978,2018. 